\title{
A novel denoising framework for cerenkov luminescence imaging based on spatial information improved clustering and curvature-driven diffusion
}

\author{
Xin Cao*, Yi Sun*, Fei Kang ${ }^{\dagger}$, Lin Wang*, Huangjian Yi*, Fengjun Zhao*, \\ Linzhi $\mathrm{Su}^{*,+,}$ and Xiaowei $\mathrm{He}^{*, \S, \uparrow}$ \\ *School of Information Science and Technology \\ Northwest University \\ Xi'an, Shaanxi 710069, P. R. China \\ ${ }^{\dagger}$ Department of Nuclear Medicine, Xijing Hospital \\ Fourth Military Medical University \\ Xi'an, Shaanxi 710032, P. R. China \\ †sulinzhi029@163.com \\ shexw@nwu.edu.cn
}

Received 29 December 2017

Accepted 18 March 2018

Published 9 April 2018

\begin{abstract}
With widely availed clinically used radionuclides, Cerenkov luminescence imaging (CLI) has become a potential tool in the field of optical molecular imaging. However, the impulse noises introduced by high-energy gamma rays that are generated during the decay of radionuclide reduce the image quality significantly, which affects the accuracy of quantitative analysis, as well as the three-dimensional reconstruction. In this work, a novel denoising framework based on fuzzy clustering and curvature-driven diffusion (CDD) is proposed to remove this kind of impulse noises. To improve the accuracy, the Fuzzy Local Information C-Means algorithm, where spatial information is evolved, is used. We evaluate the performance of the proposed framework systematically with a series of experiments, and the corresponding results demonstrate a better denoising effect than those from the commonly used median filter method. We hope this work may provide a useful data pre-processing tool for CLI and its following studies.
\end{abstract}

Keywords: Cerenkov luminescence imaging; image processing; radionuclide imaging.

\Corresponding authors.

This is an Open Access article published by World Scientific Publishing Company. It is distributed under the terms of the Creative Commons Attribution 4.0 (CC-BY) License. Further distribution of this work is permitted, provided the original work is properly cited. 


\section{Introduction}

Cerenkov Luminescence Imaging (CLI) is an emerging optical molecular imaging technology, and it is based on the Cerenkov radiation (CR), which was first discovered by Pavel Alekseyevich Cerenkov in $1934 .^{1,2}$ Simply speaking, CR is a kind of visible light with continuous spectrum, which can be emitted when a charged particle (or a particle with high energy) moves in a dielectric media with speed larger than that of light in the media. ${ }^{3}$ The first time to use CR in the optical molecular imaging filed was in 2009, when Roberson et al. utilized optical instrument (OI) to collect the CR emitted during the decay of 2-18-fluoro-D-glucose $\left({ }^{18} \mathrm{~F}\right.$ FDG), and named this technology as CLI. ${ }^{4}$ With a widely used clinical radionuclides approved by Food and Drug Administration (FDA), the CLI has built a bridge between optical molecular imaging and traditional medical nuclear imaging, and has been applied in diagnostic imaging, drug development, intraoperative guidance and endoscopic imaging successfully, and the imaging objects include not only small animals, but also human beings. ${ }^{5-10}$

As the $\mathrm{CR}$ is the secondary product during the decay of radionuclides, its intensity is extremely weak, and most of the energy is distributed in the spectrum with a wavelength below $600 \mathrm{~nm} \cdot{ }^{11,12}$ Thus, the detection of $\mathrm{CR}$ in in vivo studies is difficult because the high attenuation of CR during the transmission in biological tissues and a relatively long acquisition time is needed $(3-5 \mathrm{~min}$ in most in vivo CLI studies due to the low activity of radionuclide). ${ }^{13}$ Because the main product of nuclear decay is high-energy gamma rays, during the acquisition of $\mathrm{CR}$, these high-energy gamma rays will penetrate the biological tissue and hit the chargedcouple device (CCD) of the OI directly, resulting a number of impulse noises with very high grayscale value on the CLI images, and reducing the accuracy of the follow-up researches, such as quantitative analysis, three-dimensional reconstruction, etc. In order to suppress these noises, one method adopted in most CLI studies is the median filter algorithm. Although the median filter algorithm can remove most of the noises with an appropriate kernel size, there are still two drawbacks. First, the intensity of CL source will be reduced in the filtered image due to the features of median filter algorithm, making the image not suitable for quantitative analysis. Second, a good denoising effect corresponds to a relatively large kernel size, which would lead to the change of the shape of CL source. Besides the denoising method mentioned above, Cao et al. proposed a temporal median (TM) filter algorithm to remove the noises. The method captures a temporal sequence of CLI images (30 images) with short exposure time $(10 \mathrm{~s})$ and then a TM filter is used to smooth a temporal sequence of the image pixels. ${ }^{14}$ The animal pseudotumor experiment (the activity of radionuclide is $100 \mu \mathrm{Ci}$ ) results demonstrated that the TM filter works well, but one important thing should be noted is that in most of the in vivo studies, CL can be hardly detected during such a short exposure time because the activity of radionuclide gathered in the lesion is very low, resulting a long acquisition time. Thus, the TM filter may not be so suitable for CLI image denoising as the total acquisition time would be very long.

Here, we regard that the procedure of CLI image denoising contains two steps: the removal of noise pixels and image inpainting. As mentioned above, the CLI image is corrupted by high-value noises, so the pixels can be divided into three categories: background, signal and noise. Thus, the denoising in CLI image is to abandon the noise part. Inspired by the pattern recognition techniques applied in image processing, the clustering approach can be considered as an ideal tool. The Fuzzy C-means (FCM) technique is the basic algorithm to cluster the data into several categories and it can retain more detail information due to its utilization of memberships. ${ }^{15}$ However, FCM is sensitive to noise because it fails to discover the co-relationship among pixels without involving appropriate spatial information. ${ }^{16}$ To solve the problem, several improved techniques were proposed. ${ }^{17-19}$ Among them, the Fuzzy Local Information C-Means (FLICM) algorithm not only incorporates local spatial information but also free from artificial parameters, so it has been demonstrated as an outstanding technique both theoretically and practically. ${ }^{20}$ However, as some pixels belonging to the region of interest (ROI) on CLI image have a relatively high grayscale value, these points may be clustered to the noise part. Thus, the denoising process cannot simply abandon this part and a further process is needed for each of the clustering result. After the removal of noise pixels, there will be some pixels with the grayscale value of 0 , including some points belonging to ROI, so it is 
necessary to fulfill these points by using a proper imaging inpainting method. Simply speaking, inpainting is an interpolation problem, filling the unknown pixels with a condition to agree with the known image on the boundary. ${ }^{21}$ A commonly used method for image inpainting is to solve Laplace's equation, but it is not suitable for image for making the image over smooth, resulting in the loss of detailed information. To overcome the problem mentioned above, Chan et al. proposed an inpainting model based on the Total Variation (TV) inpainting, named CDD model. ${ }^{22}$ Compared to the TV inpainting, the CDD model can restore a single object when its disconnected remaining parts are separated far apart by inpainting domain, which will be used in our proposed denoising framework.

In this study, we proposed a novel denoising framework for CLI, which combined FLICM algorithm and CDD Model, named as FLICMCDD for short. The robustness and usability of FLICMCDD is tested via a series of experiments. Unlike traditional median filter algorithm and TM filter algorithm, the FLICMCDD can remove the high-energy rays raised noises effectively with the shape of CL source unchanged, and a relatively long exposure time ensures the detection of CL under the situation of low radioactivity in in vivo studies.

\section{Materials and Methods}

\subsection{Proposed denoising framework for CLI images}

Suppose the CLI image to be studied is $I$, and $I(i, j)$ denotes the intensity of the pixel $(i, j)$. First of all, by analyzing the histogram of CLI image to acquire the threshold value $\boldsymbol{T}$, the pixels with the value greater than $\boldsymbol{T}$ can be simply regarded as noises. For example, Fig. 1(b) shows the histogram of Fig. 1(a) while Fig. 1(a) is a CLI image, which will be used in the section of in vivo experiment. It is obviously that $\boldsymbol{T}$ should be set to the number between 40,000 and 60,000 .

After the initialization of threshold value $\boldsymbol{T}$, FLICM was performed to divide CLI image to three parts: background, signal and noise. Then, we find the isolated pixel and set the value to 0 :

$$
\begin{aligned}
& \text { If } \max _{x \in \Omega_{i j}}\{I(x)\}=0, \\
& \text { then } I(i, j)=0,
\end{aligned}
$$

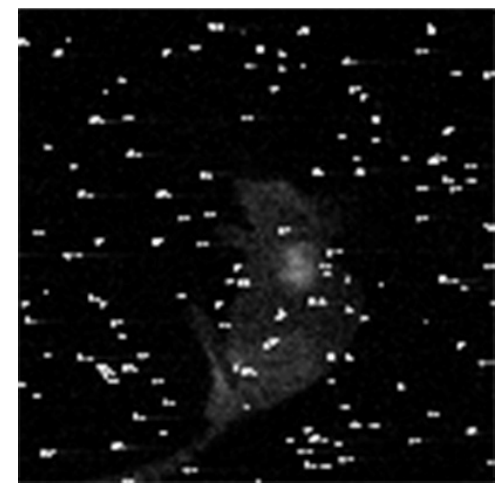

(a)

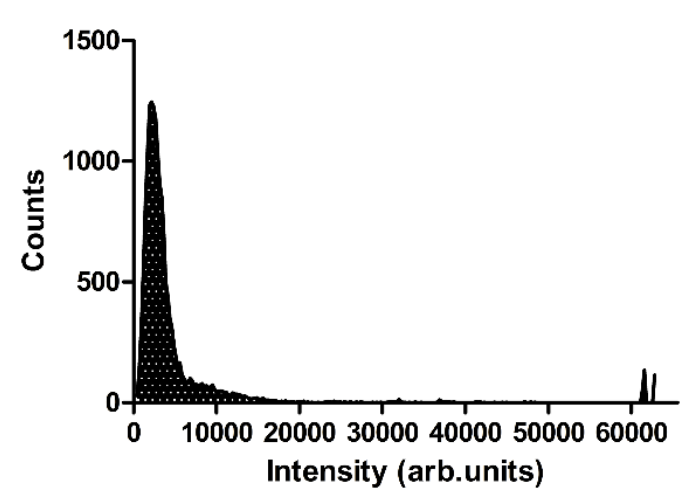

(b)

Fig. 1. (a) A CLI image of in vivo experiment and (b) the histogram of (a).

where $\Omega_{i j}$ denotes the $3 \times 3$ deleted neighborhood of $(i, j)$. It should be noted that $\Omega_{i j}$ dose not contain $(i, j)$ itself. After the removal of isolated pixels, the holes of each images are filled with the mean value of the neighbored pixels:

$$
\begin{aligned}
& \text { If } \min _{x \in \Omega_{i j}}\{I(x)\}>0 \text { and } I(i, j)=0, \\
& \text { then } I(i, j)=\operatorname{mean}_{x \in \Omega_{i j}}\{I(x)\} .
\end{aligned}
$$

It should be noted that in this study, the number of neighbored pixels was 8. Next, denoised CLI image is generated by simply overlaying the images, and then removing the pixels with the value greater than $\boldsymbol{T}$ :

$$
\begin{aligned}
& \text { If } \quad I(i, j)>\boldsymbol{T}, \\
& \text { then } \quad I(i, j)=0 .
\end{aligned}
$$

Finally, the CDD model for imaging inpainting is used. The denoising framework based on FLICMCCD is given as follows. 


\begin{tabular}{ll}
\hline Algorithm: & Denoising framework based on FLICMCDD \\
\hline Begin &
\end{tabular}

Initialization. Get the threshold value $\boldsymbol{T}$ by analyzing the histogram of CLI image.

Step 1. Dividing CLI image to three categories by using FLICM method: background, signal and noise.

Step 2. Removing the isolated pixels by using (1) for each image.

Step 3. Filling the holes of each images by using (2).

Step 4. Generating the denoised CLI image by overlaying the images.

Step 5. Removing the pixels with the value greater than $\boldsymbol{T}$ using (3).

Step 6. Using CDD model for imaging inpainting.

End

\subsection{Numerical simulation experiment}

In order to generate a simulated CLI image, Molecular Optical Simulation Environment (MOSE) software based on digital mouse atlas is utilized here. ${ }^{23} \mathrm{~A}$ point light source is subcutaneously embedded on the back of the digital mouse to mimic medical isotope and the depth of the source was about $5 \mathrm{~mm}$, as shown in Fig. 2(a). The wavelength of luminescence simulated here is set to $620 \mathrm{~mm}$, and the optical parameters of each organ are adopted from Ref. 24. Figure 2(b) is the simulated CLI image without noises, and Fig. 2(c) is the simulated CLI image after the addition of dark noises of CCD and impulse noises introduced by high-energy gamma rays. All the images are fused with mouse atlas and the above procedure was repeated five times.

\subsection{Materials and instrument for physical experiments}

The radionuclide used in this study is ${ }^{18} \mathrm{~F}$, obtained in the form of ${ }^{18} \mathrm{~F}-\mathrm{FDG}$, which is produced via a cyclotron (GE Industries Inc., USA) and the FDG reagent kit (ABX, Germany).

The OI is a home-made CLI system, it is composed of an electron multiplying CCD (EMCCD) camera (Ixon3 Ultra 897, Andor Corp), a standard prime lens (Pentax F/1.8) and a light-tight box to avoid the interface of ambient light. It should be noted that the process of imaging an object contains the collection of two images, white-light image with an integration time of $0.1 \mathrm{~s}$ and CLI image with an integration time of $3 \mathrm{~min}$, and the bin value is set to 4 . All the procedures of acquiring CLI images are repeated five times.

\subsection{Physical phantom experiment}

To investigate whether the proposed denoising framework could affect the shape of CL source, a nylon phantom-based experiment is conducted. A cubic phantom with the length of $10 \mathrm{~mm}$ is manufactured and a hole is drilled from its upper surface. Distance from the center of the hole to the side surface is $2.5 \mathrm{~mm}$. A rubber capillary tube filled with a mixture of $740 \mathrm{KBq}(20 \mu \mathrm{Ci}){ }^{18} \mathrm{~F}-\mathrm{FDG}$ is inserted into the hole. The phantom is then placed

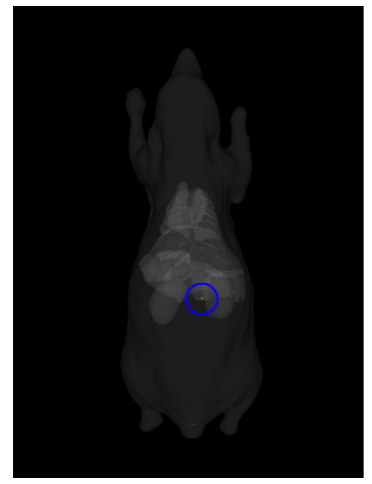

(a)

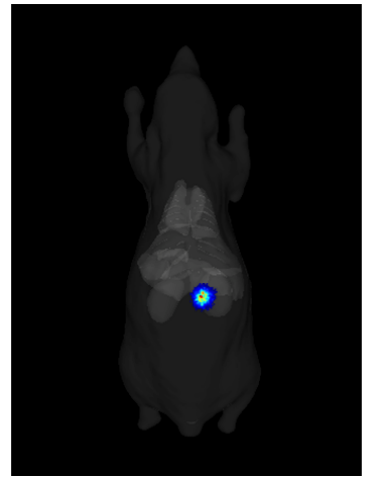

(b)

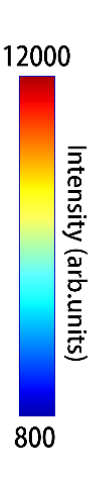

800

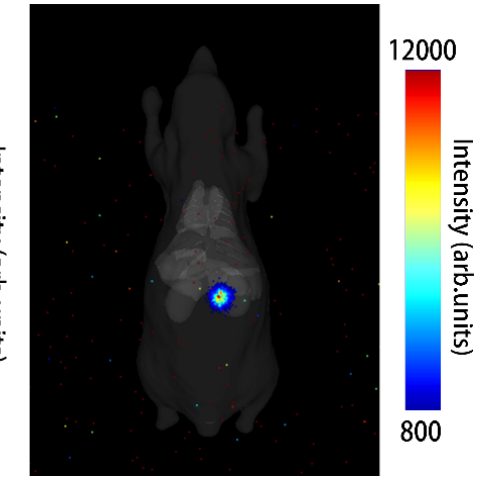

(c)

Fig. 2. Numerical Simulation of CLI image: (a) the digital mouse and CL source location, which is the red point in the blue circle; (b) simulated CLI image and (c) simulated CLI image after the addition of noises. 
in the FOV of CLI system and a white image as well as a CLI image is obtained.

\subsection{In vivo experiment}

To investigate the potential of the proposed denoising framework for biomedical applications, pseudotumor-based experiment is conducted in this section. The pseudotumor animal model is applied according to the description of our previous studies. ${ }^{5,13}$ Animal care and protocols are approved by the Fourth Military Medical University Animal Studies Committee. All animal procedures are performed under general anesthesia by inhalation of $1-2 \%$ isoflurane-oxygen mixture. A mixture is made of $50 \mu \mathrm{L}$ Matrigel (BD Biosciences) and $555 \mathrm{KBq}$ $(15 \mu \mathrm{Ci}){ }^{18} \mathrm{~F}-\mathrm{FDG}$ to form a final volume of $100 \mu \mathrm{L}$ in a microfuge tube. It is then subcutaneously injected in the left forearm of a nude mouse. The mouse is kept warm for $3 \mathrm{~min}$ until the Matrigel solidified. After the mouse is placed on a warmed platform, it is moved into the system and images were acquired.

\section{Results and Discussion}

\subsection{Numerical simulation experiment}

Denoising results by using median filter are shown in Figs. 3(a)-3(c), while Fig. 3(d) is the result by using FLICMCDD. As the kernel size of median filter can affect the denoising effect, different kernel sizes (size $=3,5$ and 7 , respectively) are used here to compare the denoising effect between median filter and FLICMCDD, while the root mean squared error (RMSE) of ROI is calculated to assess the denoising results by

$$
\operatorname{RMSE}=\sqrt{\frac{\sum_{i=0}^{n}\left(I_{o}-I_{f}\right)^{2}}{n}},
$$

where $I_{o}$ denotes the pixels of simulated CLI image without adding noises, and $I_{f}$ denotes the pixels of the filtered simulated CLI image. It is obvious that the lower RMSE value indicates a better denoising effect. Figure 3(e) is the calculated RMSE of the two methods. It is easy to find out that even the median filter can effectively remove noises, large

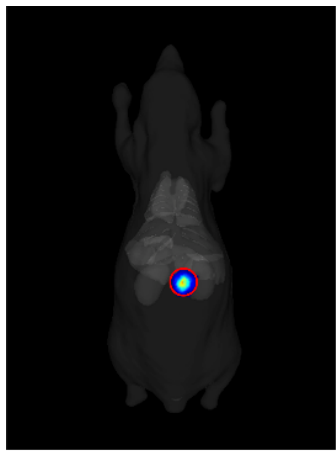

(a)

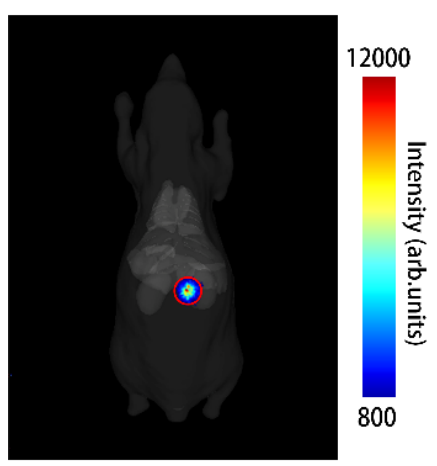

(d)

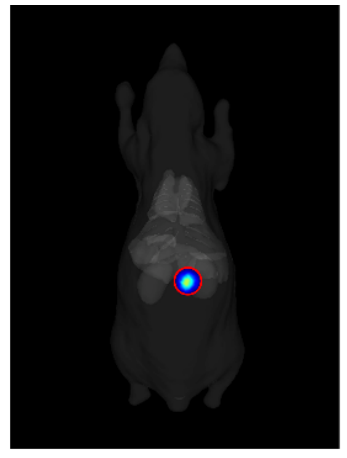

(b)
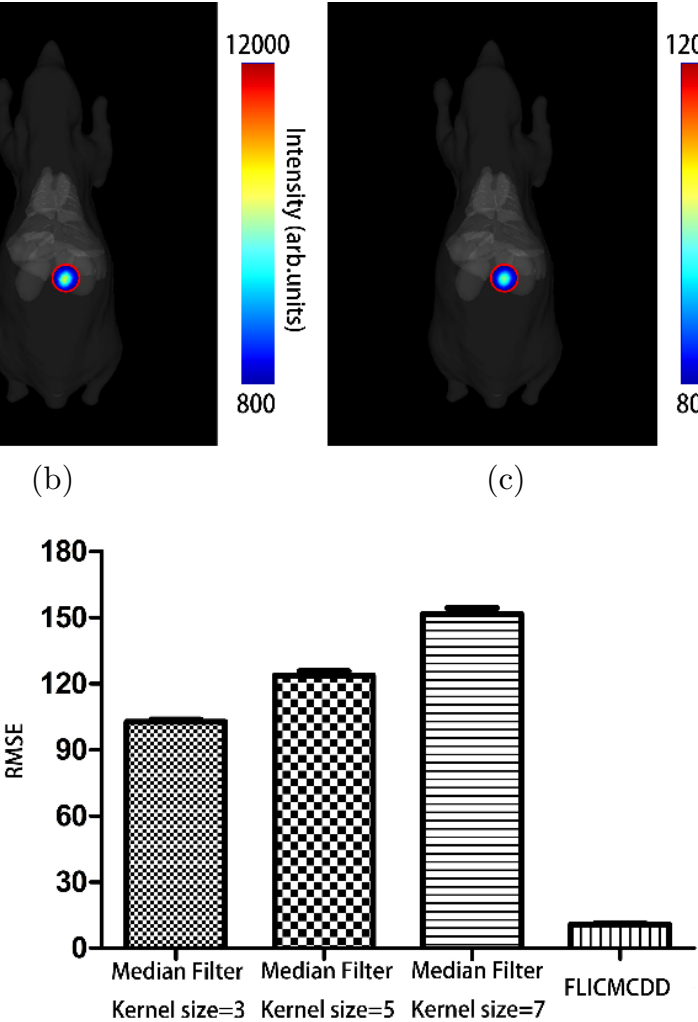

(e)

Fig. 3. A comparison of RMSE value between median filter and FLICMCDD: (a)-(c) results of median filter with different kernel sizes (size $=3,5$ and 7, respectively); (d) result of FLICMCDD and (e) comparison of RMSE value of ROI for the FLICM and median filter. 
RMSE values would also emerge, and the larger the kernel size used, the larger the RMSE value. As to the result of using FLICMCDD, a relatively good denoising effect is obtained here with a low RMSE value (lower than 15), demonstrated that the proposed method can keep the pixel intensity nearly unchanged.

\subsection{Physical phantom experiment}

Fusion image of the original CLI image and whitelight image is shown in Fig. 4(a). The result of FLICMCDD is shown in Fig. 4(b), while that of median filter is shown in Fig. 4(c). It should be noted that the kernel size of median filter used here is 5. A profile is extracted along with a red line depicted in Figs. 4(a)-4(c), and depicted in Fig. 4(d). It is obvious that the profile of original CLI image is similar to that of the processed image by using FLICMCDD, while a relatively big difference exists between the profile of original CLI image and denoised image by using the median filter.

To further investigate whether the proposed denoising framework can maintain the shape of
CL source, structural similarity index (SSIM) is used here:

$$
\operatorname{SSIM}\left(I_{1}, I_{2}\right)=\frac{\left(2 \mu_{1} \mu_{2}+c_{1}\right)\left(2 \sigma_{1,2}+c_{2}\right)}{\left(\mu_{1}^{2}+\mu_{2}^{2}+c_{1}\right)\left(\sigma_{1}^{2}+\sigma_{1}^{2}+c_{2}\right)},
$$

where $\mu_{1}$ denotes the mean intensity value of image $I_{1}, \mu_{2}$ denotes the mean intensity value of image $I_{2} \cdot \sigma_{1}^{2}$ is the variance of image $I_{1}$, while $\sigma_{2}^{2}$ denotes the variance of image $I_{2} \cdot \sigma_{1.2}$ is the covariance between $I_{1}$ and $I_{2} \cdot c_{1}=\left(k_{1} L\right)^{2}$ and $c_{2}=\left(k_{2} L\right)^{2}$ are two variables to stabilize the division with weak denominator, where $L$ is the dynamic range of the pixel values (the value is 16 in this study). $k_{1}=0.01$ and $k_{2}=0.03$ by default. It should be noted that larger the SSIM, the higher the degree of similarity of the two images.

Result of calculated SSIM of is shown Fig. 4(e), it can be concluded that the shape of CL source can keep very well by using FLICMCDD, compared to the result of median filter.

\subsection{In vivo experiment}

Figure 5(a) shows the white-light image of the mouse acquired by CLI system, and red circle

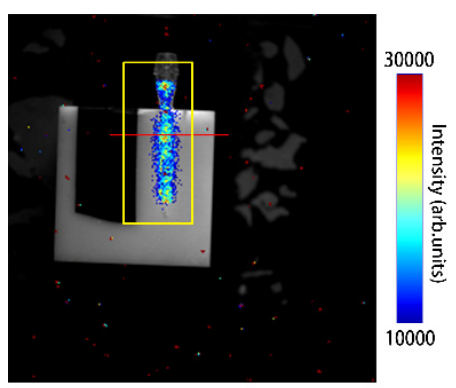

(a)

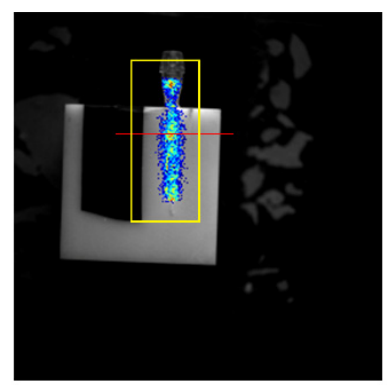

(b)

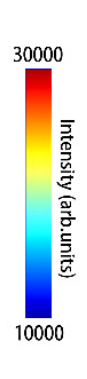

.... None

---- FLICMCDD

....-.- Median Filter

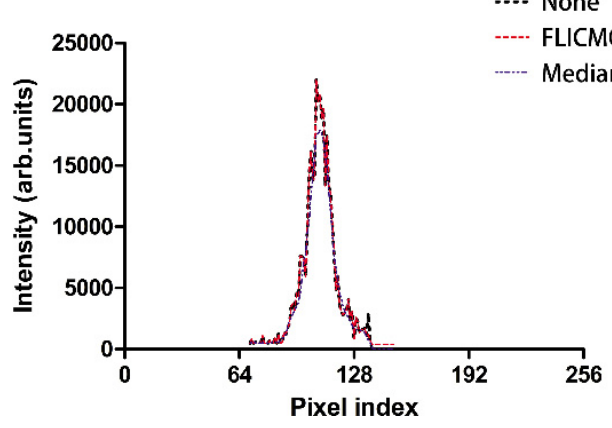

(d)

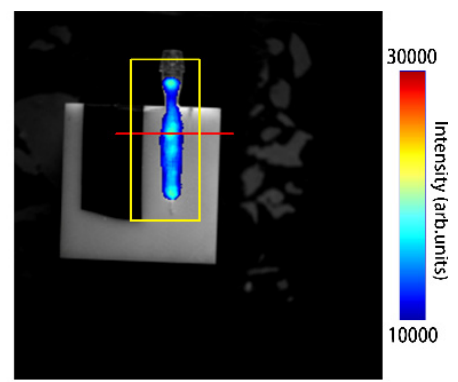

(c)

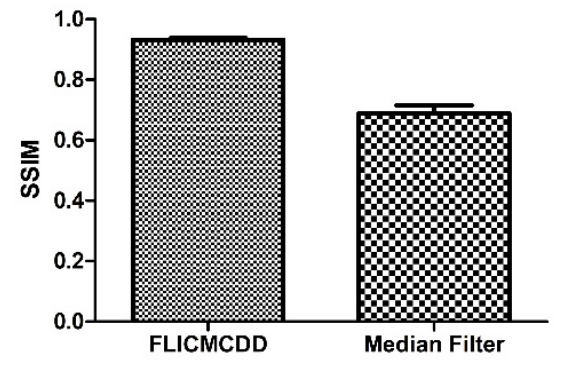

(e)

Fig. 4. Result of physical phantom experiment: (a) original CLI image; (b) denoised CLI image by using FLICMCDD; (c) denoised CLI image by using median filter; (d) quantitative line profile (red line in (a), (b) and (c), respectively) and (e) comparison of SSIM (yellow rectangle) for FLICMCDD and median filter. 


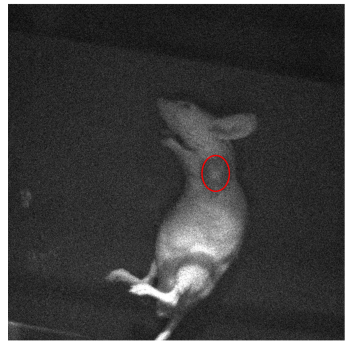

(a)

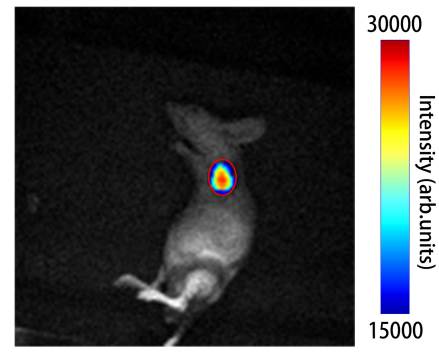

(d)

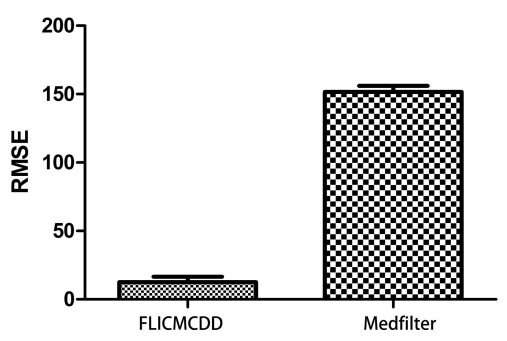

(f)

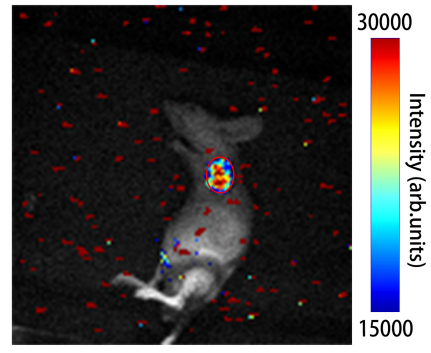

(b)

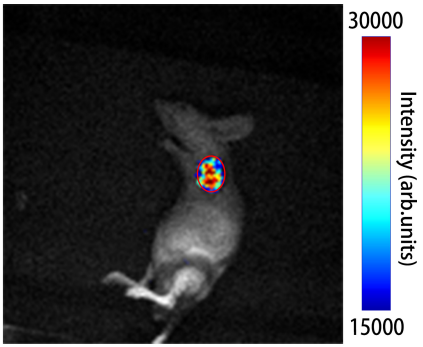

(c)

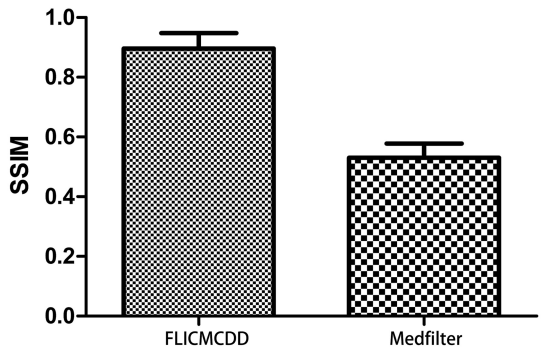

(e)

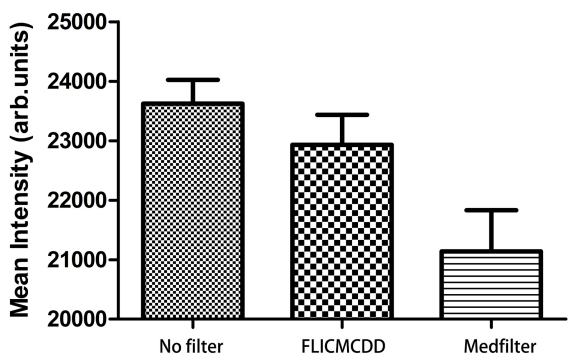

(g)

Fig. 5. Result of the in vivo experiment: (a) white-light image of mouse, the pseudotumor area is outlined in red circle; (b) original CLI image; (c) denoised CLI image by using FLICMCDD; (d) denoised CLI image by using median filter; (e) comparison of SSIM (red circle) for FLICMCDD and median filter; (f) comparison of RMSE for FLICMCDD and median filter and (g) mean intensity of ROI of (b), (c) and (d), respectively.

depicts the location of the subcutaneous pseudotumor. CLI image is shown in Fig. 5(b), and Fig. 5(c) is the denoised image by using FCLICMCDD, while the result of using median filter is shown in Fig. 5(d). The kernel size of median filter is 5. SSIM value, RMSE value and mean intensity of ROI (the region of red circle) are calculated and shown in Figs. $5(\mathrm{e})-5(\mathrm{~g})$. All the results indicate that little difference remains between original CLI image and denoised image by using FLICMCDD, with the impulse noises are removed clearly, which demonstrates that the proposed framework works well for the real biological applications.

\section{Conclusion}

In conclusion, a novel denoising framework for CLI image is proposed in this work, which can remove the random impulse noises introduced by highenergy gamma rays generated during the decay of radionuclide. A series of experiments are designed and conducted to evaluate the effect of the proposed framework. Results demonstrated that, compared to the commonly used median filter, the proposed framework works well with the preservation of the shape of CL source, as well as the intensity. We hope this work may provide a useful data pre-processing tool for CLI and its following studies.

\section{Acknowledgments}

This work was supported by the Program of the National Natural Science Foundation of China under Grant Nos. 61701403, 61601363, 11571012, 61372046 and 61640418, the Natural Science Basic 
Research Plan in Shaanxi Province of China under Grant Nos. 2017JQ6006 and 2017JQ6017.

\section{References}

1. Y. Xu, H. Liu, Z. Cheng, "Harnessing the power of radionuclides for optical imaging: Cerenkov luminescence imaging," J. Nucl. Med. 52, 2009-2018 (2011).

2. P. A. Čerenkov, "Visible radiation produced by electrons moving in a medium with velocities exceeding that of light," Phys. Rev. 52, 378-379 (1937).

3. A. Ruggiero, J. P. Holland, J. S. Lewis et al., "Cerenkov luminescence imaging of medical isotopes," J. Nucl. Med. 51, 1123-1130 (2010).

4. R. Robertson, M. S. Germanos, C. Li et al., "Optical imaging of cerenkov light generation from positron-emitting radiotracers," Phys. Med. Biol. 54, N355-N365 (2009).

5. X. Cao, X. Chen, F. Kang et al., "Performance evaluation of endoscopic cerenkov luminescence imaging system: In vitro and pseudotumor studies," Biomed. Opt. Express 5, 3660-3670 (2014).

6. X. Cao, Y. Zhan, X. Cao et al., "Harnessing the power of cerenkov luminescence imaging for gastroenterology: Cerenkov luminescence endoscopy," Curr. Med. Imag. Rev. 13, 50-57 (2017).

7. Z. Hu, J. Liang, W. Yang et al., "Experimental cerenkov luminescence tomography of the mouse model with spect imaging validation," Opt. Express 18, 24441-24450 (2010).

8. C. Li, G. S. Mitchell, S. R. Cherry, "Cerenkov luminescence tomography for small-animal imaging," Opt. Lett. 35, 1109-1111 (2010).

9. A. E. Spinelli, M. Ferdeghini, C. Cavedon et al., "First human cerenkography," J. Biomed. Opt. 18, 020502 (2013).

10. D. L. J. Thorek, C. C. Riedl, J. Grimm, "Clinical cerenkov luminescence imaging of f-18-fdg," J. Nucl. Med. 55, 95-98 (2014).

11. X. Cao, X. Chen, F. Kang et al., "Sensitivity improvement of cerenkov luminescence endoscope with terbium doped gd2o2s nanoparticles," Appl. Phys. Lett. 106, 213702 (2015).

12. G. S. Mitchell, R. K. Gill, D. L. Boucher et al., "In vivo cerenkov luminescence imaging: A new tool for molecular imaging," Philos. Trans. R. Soc. Lond. A, Math. Phys. Eng. Sci. 369, 4605-4619 (2011).

13. X. Cao, X. Chen, F. Kang et al., "Intensity enhanced cerenkov luminescence imaging using terbiumdoped gd2o2s microparticles," ACS Appl. Mater. Interfaces 7, 11775-11782 (2015).

14. X. Cao, Y. Li, Y. Zhan et al., "Removing noises induced by gamma radiation in cerenkov luminescence imaging using a temporal median filter," Biomed. Res. Int. 2016, 7948432-7948432 (2016).

15. J. C. Bezdek, Pattern Recognition with Fuzzy Objective Function Algorithms, Plenum Press, New York (1981).

16. P. Wang, H. L. Wang, A modified FCM algorithm for MRI brain image segmentation, Int. Seminar Future BioMedical Information Engineering, 2008. FBIE'08, pp. 26-29, IEEE (2008).

17. Y. A. Tolias, S. M. Panas, "Image segmentation by a fuzzy clustering algorithm using adaptive spatially constrained membership functions," IEEE Trans. Syst. Man Cybern. A, Syst. Humans 28, 359-369 (1998).

18. M. Gong, L. Su, M. Jia et al., "Fuzzy clustering with a modified mrf energy function for change detection in synthetic aperture radar images," IEEE Trans. Fuzzy Syst. 22, 98-109 (2014).

19. M. Krinidis, I. Pitas, "Color texture segmentation based on the modal energy of deformable surfaces," IEEE Trans. Image Process. 18, 1613-1622 (2009).

20. S. Krinidis, V. Chatzis, "A robust fuzzy local information c-means clustering algorithm" IEEE Trans. Image Process. 19, 1328-1337 (2010).

21. T. F. Chan, J. H. Shen, "Mathematical models for local nontexture inpaintings," SIAM J. Appl. Math. 62, 1019-1043 (2002).

22. T. F. Chan, "Nontexture inpainting by curvaturedriven diffusions," J. Vis. Commun. Image Represent. 12, 436-449 (2001).

23. S. H. Ren, X. L. Chen, H. L. Wang et al., "Molecular optical simulation environment (mose): A platform for the simulation of light propagation in turbid media," PLoS One 8, 11 (2013).

24. K. Liu, Y. Lu, J. Tian et al., "Evaluation of the simplified spherical harmonics approximation in bioluminescence tomography through heterogeneous mouse models," Opt. Express 18, 20988-21002 (2010). 